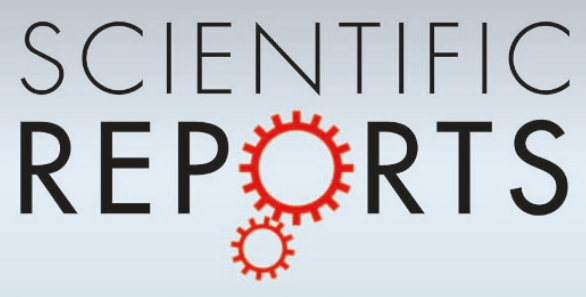

OPEN

SUBJECT AREAS:

APPLIED PHYSICS

FLUID DYNAMICS

MECHANICAL ENGINEERING

Received

10 December 2013

Accepted

5 March 2014

Published

30 April 2014

Correspondence and requests for materials should be addressed to

J.-C.B. (jean-

christophe.baret@ds.

mpg.de)

* These authors contributed equally to this work.

$†$ CNRS, Univ. Bordeaux, CRPP, UPR 8641, 33600 Pessac (France).

\section{The Microfluidic Jukebox}

\author{
Say Hwa Tan*, Florine Maes*, Benoît Semin, Jérémy Vrignon \& Jean-Christophe Baret†
}

Max Planck Institute for Dynamics and Self-organization - Droplets, Membranes and Interfaces, Am Fassberg 17, D-37077 Goettingen, Germany.

Music is a form of art interweaving people of all walks of life. Through subtle changes in frequencies, a succession of musical notes forms a melody which is capable of mesmerizing the minds of people. With the advances in technology, we are now able to generate music electronically without relying solely on physical instruments. Here, we demonstrate a musical interpretation of droplet-based microfluidics as a form of novel electronic musical instruments. Using the interplay of electric field and hydrodynamics in microfluidic devices, well controlled frequency patterns corresponding to musical tracks are generated in real time. This high-speed modulation of droplet frequency (and therefore of droplet sizes) may also provide solutions that reconciles high-throughput droplet production and the control of individual droplet at production which is needed for many biochemical or material synthesis applications.
T he development of gaming and entertaining strategies in Science and Technology is emerging: as an example, recent approaches such as biotic games make use of manipulation of biological material in microfluidic platforms to provide novel gaming environments ${ }^{1}$. The interest of such an approach is two fold: First the developments of gaming and entertaining strategies might point towards novel industrial applications. Second, it brings the technology to the public outside of the scientific or technology field. A very striking example of the relevance of the concept is the scientific success of the game Foldit ${ }^{2}$ : crowd-sourcing was used to solve the crystal structure of a protein, by players who had no prior scientific training ${ }^{2}$. Extending this concept to other systems is therefore of interest and in this context, music has a real potential to bridge Science and Society. As an art, music has already benefited from the progress of Science and Technology. Sounds can now be electronically generated and recorded leading to the development of novel instruments and the production of music at the industrial scale. These novel instruments complement the original instruments in which music is generated by mechanical vibrations.

Music combines entertainment and the need for reliability in controlled switching. Virtuosity in playing music is determined by the capacity to reliably control flows and vibrations to generate sounds with well defined frequencies and switch quickly between them. In addition, the analytical capabilities of the human ear and brain are tremendous. The power of the ears spans several order of magnitude in intensities and frequencies ranging from $20 \mathrm{~Hz}$ to $20 \mathrm{kHz}$. Well trained or professional musicians can distinguish between minute frequency shifts with an almost incredible level of accuracy (down to $1 \mathrm{~Hz}$ on a $440 \mathrm{~Hz}$ signal). The post-processing of the signals by the brain also allows every individual to be sensitive to fluctuations in rhythms ${ }^{3}$. These fantastic analytical capabilities of the human ears have been used in submarines ('oreilles d'or') in the military context; nowadays, acoustic emissions are also used to monitor processes in chemical engineering ${ }^{4}$. Music therefore shares a number of features and requirements of microtechnology, among them are control, switching and sensing. Investigating microfluidics through sound production is therefore relevant as an entertaining concept but at the same time will have implications for the development of novel instruments or methods for quality control and process monitoring in fields linked to micro-technology. Microfluidics, sound, and music are already linked together by the technology of Surface Acoustic Waves actuation ${ }^{5,6}$ or the control of flow by sound and pressure waves, as demonstrated recently ${ }^{7}$.

We consider here the reverse problem, namely the generation of music and sound-tracks by microfluidic flow. For about two decades, microfabrication offers tools, methods and systems to create microfluidic platforms usable for the miniaturization and automation of biological assays ${ }^{8-12}$. A significant increase of throughput, analytical quantitativity and sensitivity and reduction of cost of assays is obtained when using the microfluidic formats ${ }^{9,13-15}$. Among the most successful approaches - both in research and industrial applications - is droplet-based microfluidics ${ }^{16}$. It is a very attractive tool due to the possible universality of the principle; by encapsulating single biological components, such as cells, viruses, or genes in droplets, assays can be performed inside droplets microcompartment transported through microchannels by an immiscible oil. While the carrier liquid drives the droplets according to a pre-programmed scheme, additional controls are implemented to selectively actuate 
individual droplets. The external control by electric field offers the most interesting opportunities since single events can be controlled among the droplet population at a high throughput $(z 1 \mathrm{kHz})$, for selective fusion ${ }^{17-19}$ and sorting of hits as in Fluorescent Activated Cell Sorting ${ }^{13,18,20}$. External control might in some cases be required at the very moment of droplet production. Such a control has been demonstrated with pneumatic valves ${ }^{21}$ or ferrofluids and magnetic fields ${ }^{22}$ but these methods fail to provide the tools to control single droplet while maintaining an overall high-throughput. We proposed a system solving these limitations based on electrocapillary actuation of droplets ${ }^{23}$, going beyond the systems demonstrated up-to-date ${ }^{24}$. While such systems will find applications in biology or material sciences, we demonstrate here a new application of droplet-based microfluidics targeting entertainment through music.

\section{Results}

We use a droplet-based microfluidics system to generate water-in-oil $(\mathrm{W} / \mathrm{O})$ droplets at a flow focusing junction ${ }^{25}$ (Figure 1). In the pure hydrodynamic case, the droplet size is determined by a combination of viscosities, surface tensions, flow rates and typical channel dimensions ${ }^{26,27}$ following non trivial scalings. For fixed flow conditions, the monodispersity of the emulsion generated is also known to be a function of the droplet production regime (squeezing, dripping, jetting $)^{28,29}$. For example, droplets formed in the dripping regime are highly monodispersed and droplets formed in the jetting regime show an increase in polydispersity. We set up the flow rate to be in the dripping regime to produce monodisperse droplets. The droplets being monodisperse, mass conservation simply relates the droplet volume $V$ to the frequency of production $f$ and the dispersed phase flow rates $Q_{d}: f=Q_{d} / V$ corresponding to a well defined frequency of production. Varying the oil and water flow rates from 250 to $1600 \mu \mathrm{L} / \mathrm{hr}$ and 75 to $200 \mu \mathrm{L} / \mathrm{hr}$ we obtained frequencies ranging from $150 \mathrm{~Hz}$ to $600 \mathrm{~Hz}$.

Classically, droplet-based microfluidics is used to generate droplets at frequencies of $\sim 1 \mathrm{~Hz}$ to $20 \mathrm{kHz}$, which covers the range of frequencies a human ear detects. We propose a transducing method to convert droplet production into sound tracks. We use fluorescently labeled droplets as the dispersed phase. The microfluidic chip is interfaced on an optical setup adapted from those used for dropletbased fluorescence detection ${ }^{20,30}$ (Figure 1(a)). In brief, a laser is focused in the channel through a microscope objective and the epifluorescence from the droplets is detected on a photomultiplier tube (PMT). The electrical signal from the PMT output is directly recorded on a computer using a standard sound card: we therefore use the photomultiplier tube as a transducer. We use a typical droplet production frequency of the order of $200 \mathrm{~Hz}$, much smaller than the $22 \mathrm{kHz}$ sampling rate of our sound card and the $20 \mathrm{kHz}$ cutoff frequency of our PMT. The PMT gain is tuned to have fluorescence amplitude smaller than $0.4 \mathrm{~V}$ to avoid sound saturation (Figure 1(f-h)).

Using simple signal processing tools, we determine the frequency of droplet production based on the fluorescent signal. The fundamental frequency of droplet production obtained via the Fast Fourier Transform of the PMT signal is in the range $150-700 \mathrm{~Hz}$, depending on the flow conditions (in agreement with the frequency determined by image processing of the high-speed videos and existing literature $\left.{ }^{26}\right)$. The weight of the harmonics in the FFT depend on the actual shape of the profile, for example, the size of the laser spot relative to droplet size and spacing. When the laser spot is smaller than the droplet size (as in the case of our experiments), the on/off states in the time trace are given by the ratio of droplet size to dropletdroplet distance. Therefore the weight of the harmonics is also a function of the flow conditions.

Generating a note and subsequently the series of 8 notes on the scale requires the control of droplet frequency by steps logarithmically spaced (the ratio between two successive half-tons is $2^{1 / 12}$, for equal temperament used in the following or close for the just intonation, see Supplementary Table $1^{31}$ ). A straightforward solution is to change the flow conditions. However, as sharp transitions between notes are required to play music $(\ll 1 s)$, changing flow conditions is

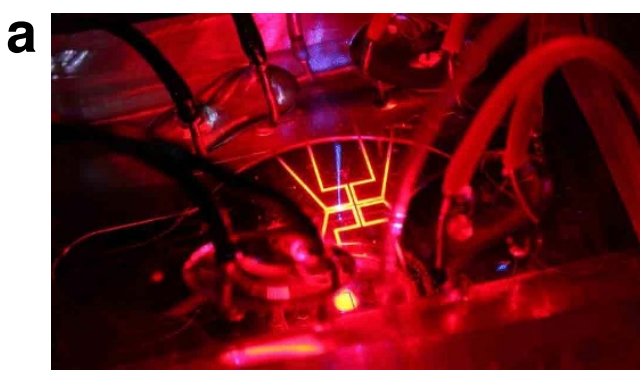

b

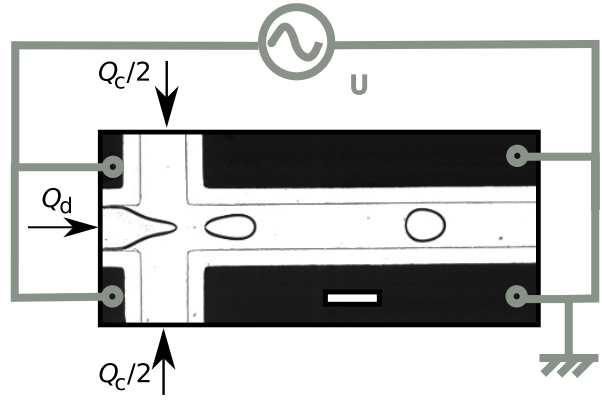

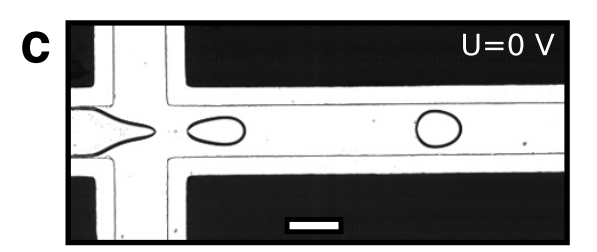

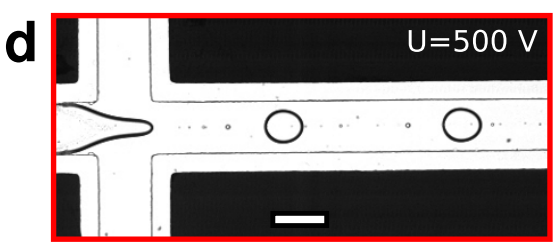

e

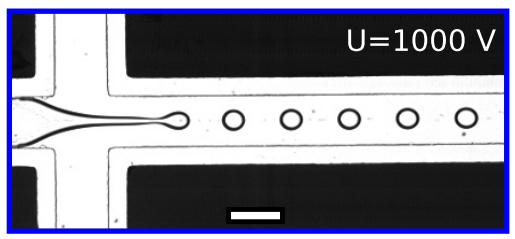

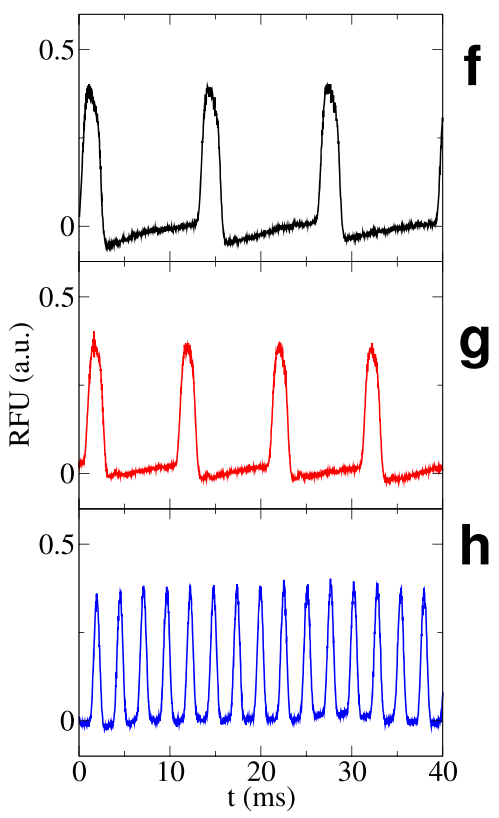

Figure 1| Concept for the microfluidic Jukebox: (a) Microfluidic chip as interfaced on the microscope. The yellow reflection shows the electrodes patterned around a flow focusing junction. The blue line in the middle is the $473 \mathrm{~nm}$ laser scattered by the PDMS. The corresponding optical setup is described as Supplementary Figure 1. (b) Detail on the fluidic and electrical connections at the flow focusing junction. Optical micrograph of the chip recorded using the high-speed camera in bright field for three values of applied voltage $U$ (c,d,e). The voltage is applied on the chip between the downstream and upstream pairs of electrodes. Corresponding droplet fluorescence signal recorded through the sound card (f,g,h). Each peak corresponds to a droplet flowing through the laser. The signal is high-pass filtered by the sound card at $50 \mathrm{~Hz}$ leading to the base line modulation. The corresponding videos are available as Supplementary Materials. 
not possible due to the slow mechanical response of the system $(>1 \mathrm{~s})$, even using optimised state-of-the-art pressure controller ${ }^{32}$. The switching between frequencies is then a key to be controlled and only a few methods previously described would provide a sufficiently fast switching to produce notes at the speed of a musical tracks ${ }^{21}$. We will show here that electrical control of droplet production ${ }^{23}$ does provide such tools and means, with very high-throughput switching capabilities.

Frequency range and musical notes. We use a new type of principle to modulate droplet size (or frequency), based on electric actuation during droplet production ${ }^{23}$. The use of electric field for droplet actuation in microfluidics is fast (with submillisecond response time), as demonstrated for droplet sorting ${ }^{20}$ or droplet controlled coalescence $^{33}$. Keeping all flow conditions constant, the interfacial properties of liquid-liquid interfaces are modulated by electric fields ${ }^{23,34}$. The electrical Maxwell stress acts as an interface contribution resulting in an effective interfacial tension function of the applied field. The droplet size in flow focusing (and hence the frequency of the fluorescent signal) is a function of the applied voltage and varies over more than a factor of two over the accessible voltage range which provides the tools to scan the whole ton scale (Figure 2).

We record the time signal of the fluorescence as a function of applied voltage and post-process the signal by Fourier Transform. Data showing the stability of the frequency as a function of time for fixed flow and voltage conditions are displayed on Figure 2. For $Q_{c}=$ $270 \mu \mathrm{L} / \mathrm{hr}, Q_{d}=85 \mu \mathrm{L} / \mathrm{hr}$ and $u=0.05 \mathrm{~V}$ or $0.845 \mathrm{~V}$, we generate a $\mathrm{C}$ or a $\mathrm{G}$ and maintain it for several seconds. We observed a long term variability of the droplet frequency (Supplementary Figure 3), linked to the mechanical stability of the syringe pumps. The evolution of the signal in the frequency space as a function of applied voltage are displayed in Figure 2. To calibrate the system (for example for different flow rate conditions) the fundamental frequency in the FFT signal is measured (see Figure 4$)$. At low voltages $(0.01<u<0.8 \mathrm{~V})$ the frequencies are varying between $180 \mathrm{~Hz}$ and $300 \mathrm{~Hz}$; at the highest voltage $(1<u<2.15 \mathrm{~V})$ the frequency reaches values between $600 \mathrm{~Hz}$ and $700 \mathrm{~Hz}$. The plateau corresponds to a region where droplets are produced in a jetting regime with very weak variation of size $\mathrm{s}^{23}$. These two parts are separated by a fast transition at $0.8<u$ $<1 \mathrm{~V}$ where the droplet sizes vary steeply as a function of applied voltage. Applying voltages by increments of $0.01 \mathrm{~V}$ or randomly, gives the same curve indicating that the system has virtually no hysteresis in the actuation, leading to a versatile and robust actuation. The stability of a given frequency is however fluctuating over large period of time leading up to about $10 \%$ over $2000 \mathrm{~s}$ (Supplementary Figure 3) The results are also reproducible from chip to chip with small variations in the shape of the curves linked to microfabrication variability.

These measurements correspond to the calibration of the system or the equivalent tuning of an instrument and give the relationship between voltages and notes. The scale is then generated by applying the corresponding voltages and our instrument covers more than one octave in frequency (depending on flow conditions). Of course, in order to obtain reliable results, the calibration is run before the music is played in order to reduce the detuning due to the mechanical instability of the pumps (See Supplementary Figure 3). a
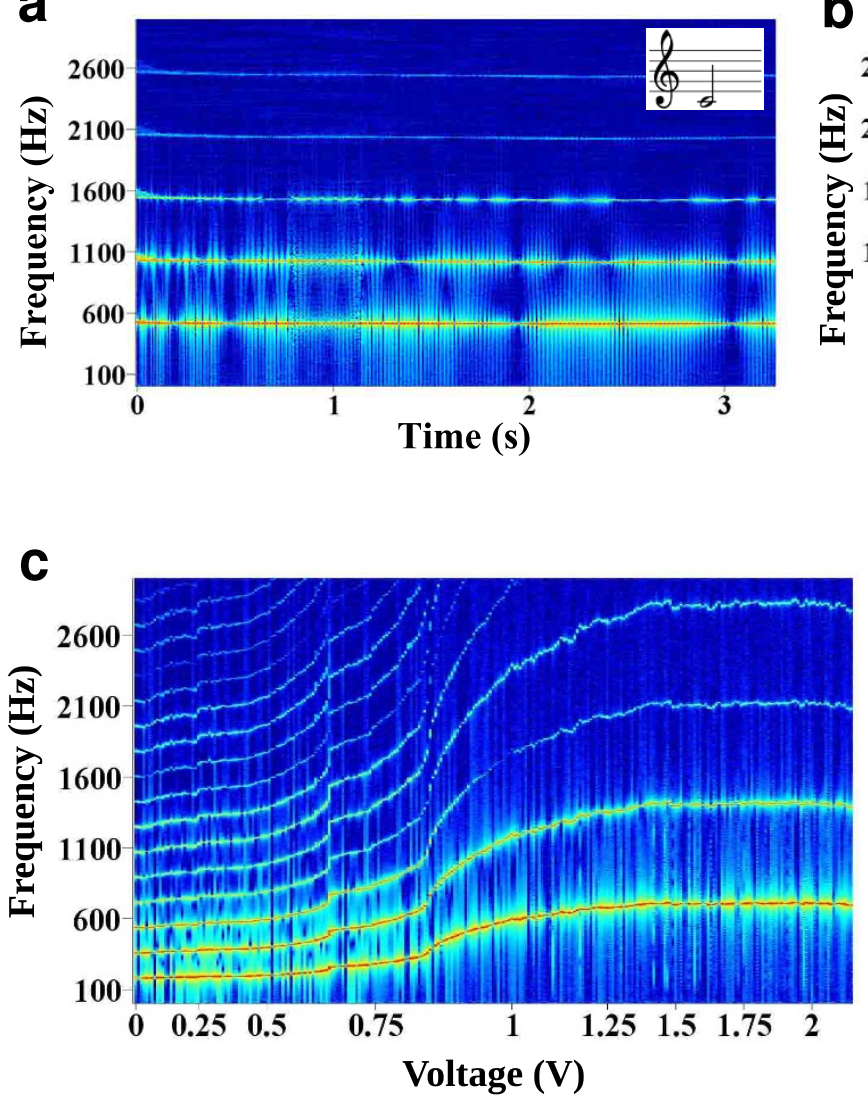

b
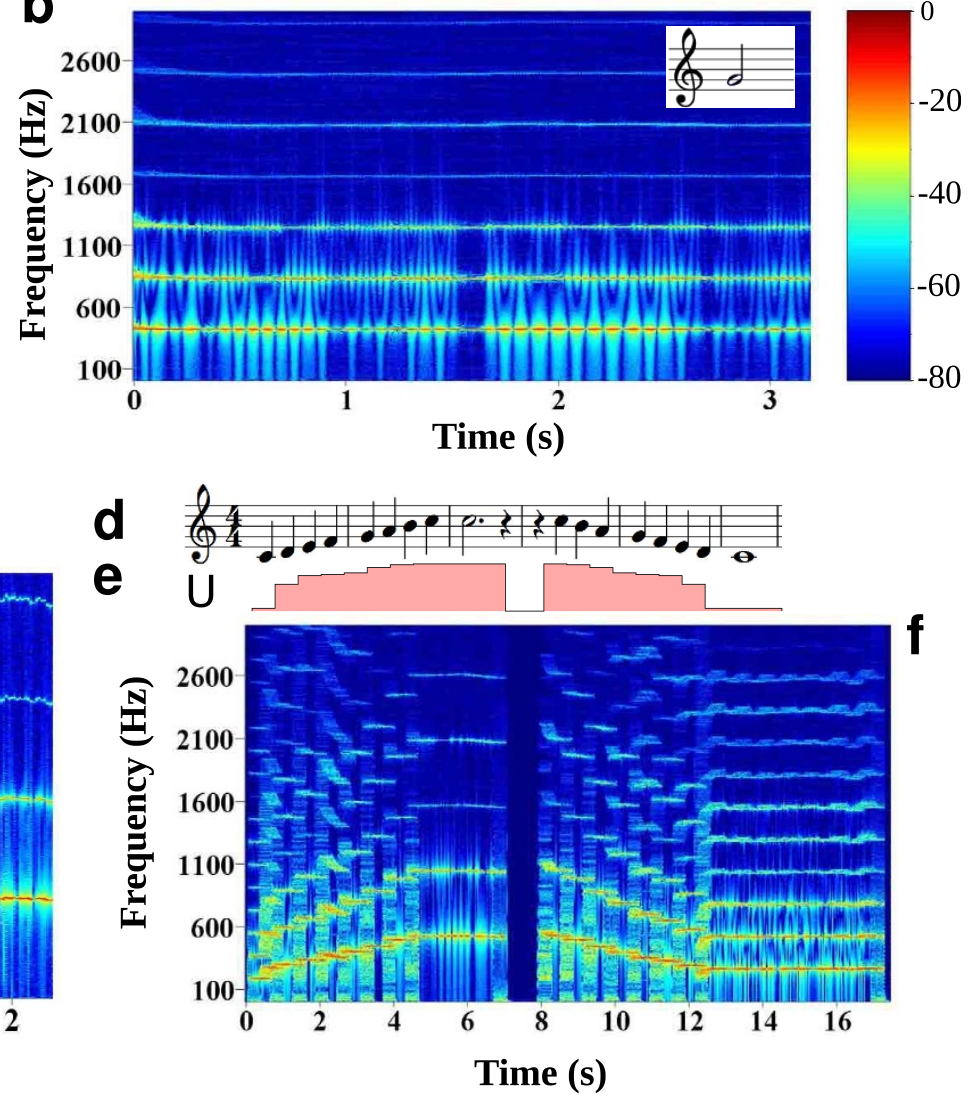

Figure $2 \mid$ Generation of musical notes by the control of droplet production frequency. Fixing the voltage across the electrodes, a well defined stable frequency is reached (example of a C (a) and a $\mathrm{G}(\mathrm{b})$ ). The frequency is a function of the applied voltage (c). This corresponds to the calibration of our musical instrument (see Supporting Information). The shape of the calibration curve depends on the flow conditions $\left(\mathrm{Here} Q_{c}=270 \mu \mathrm{L} / \mathrm{hr}\right.$, $Q_{d}=85 \mu \mathrm{L} / \mathrm{hr}$ ). Specific frequencies can be reached to play the whole scale (d) using a control of the applied voltage (e). The time trace of the frequency spectrum shows both the fundamental frequency and the harmonics in the signal ( $\mathrm{f}$ ). 
Speed response and switching between notes. In a second step, we consider the response time of the system to the electrical actuation as playing music requires fast switching capabilites (subsecond). The analysis is performed by applying an amplitude modulation (AM) of the $50 \mathrm{kHz}$ sinusoid wave by square wave of frequencies $F_{m}$ (typically 10 to $1000 \mathrm{~Hz}$ ). In order to characterize and determine the speed of the response accurately, the high speed camera is synchronized with the modulation voltage. The videos are then analyzed using simple image processing tool (Matlab/Scilab), for example by drawing space-time diagrams of the symmetry line along the flow direction. The black droplet interface appears here as white traces (Figure 3). The diagram shows the change of droplet frequency (spacing between the parallel lines) and the speed of droplet (slope of the lines).

The flow rates are fixed at $75: 500 \mu \mathrm{L} / \mathrm{hr}$ for the dispersed and continuous phase respectively resulting in a droplet formation frequency of the order $250 \mathrm{~Hz}$. The depth of the AM signal is varied between either $0 \mathrm{~V}$ or $1000 \mathrm{~V}$ at a fixed frequency of $40 \mathrm{~Hz}$ (Figure 3(a)). The results shows that when the AM signal is "on", the system responds in less than $25 \mathrm{~ms}$ (for the formation of smaller droplets) with the formation of a jet. This process is highly repeatable as shown in the space-time diagram. Such a high speed response is already sufficient to switch between notes to play music. We have further tested the actuation response time for higher flow rates in order to determine the limits of our actuation mechanism. The flow rates for the dispersed and continuous phase fluids are now fixed at $200: 1600 \mu \mathrm{L} / \mathrm{hr}$ (DP:CP) and the voltage is modulated between $U$ $=0$ and $500 \mathrm{~V}$. Increasing the flow rates allow droplets to be generated at faster rates $(500 \mathrm{~Hz})$ and we consider switching within the dripping regime (No transition to the jetting regime). The space-time diagram shows that a $40 \mathrm{~Hz}$ modulation is easily reached and the maximum frequency for which a modulation of size is obtained is about $400 \mathrm{~Hz}$ (Figure 3(b,c)). Again here, the measurement of the response time is limited by the frequency of droplet production without field $(500 \mathrm{~Hz})$. To our knowledge only electroactuation provides tools to reach reliably such short response times. With electric fields, droplet sorting can be performed at rates of up to 2000 droplets per second ${ }^{20}$; we believe that the system is capable of achieving a faster response time if other experimental parameters such as geometry of the channels and the fluids used are further optimized. However, the current obtained response time of about $2.5 \mathrm{~ms}$ is more than sufficient in the production of musical notes in our current set of experimental conditions, but is also usable for other applications where single droplet actuation is required at high throughput.

In these experiments, the response time is at least of order of the droplet production frequency. One limitation for the switching speed would be the electrical response of the system. We have modelled our chip by an equivalent electrical circuit ${ }^{23}$ as a high-pass filter. In the switching experiments, modulating a $f=50 \mathrm{kHz}$ sine wave by a square function of much lower frequency $\left(F_{m}<1 \mathrm{kHz}\right)$, the frequency spectrum of the signal will have components at frequencies $f \pm n \times F_{m}$ ( $n$ being integer) which are all above the cutoff frequency of our high-pass filter (at least for $n<10$ ). All these components are amplified and the voltage at the tip follows the applied voltage instantaneously.

Therefore we argue that the speed response of the system relies on the response of a capillary structure to a change of surface tension. Balancing the viscous drag by the capillary forces leads a time-scale

$$
\tau_{c a p} \sim \frac{\eta R}{\gamma}
$$

where $R \sim 10 \mu \mathrm{m}$ is the typical size of a droplet or the typical diameter of a jet, $\eta \sim 30 \mathrm{mPas}$ the oil viscosity and and $\gamma \sim$ $5 \mathrm{mN} / \mathrm{m}$ the oil water surface tension in the presence of surfactant. $\tau_{c a p} \sim 60 \mu$ s corresponds to the flow response to modification of $\gamma$ which occurs throught the Maxwell stress. This sets a limit of actuation frequency to about $10 \mathrm{kHz}$. However, another time-scale arises from the flow conditions. For a constant flow rate the volume $V$ mobilized to create a jet of about $R_{j e t} \sim 10 \mu \mathrm{m}$ in radius and of length $L_{j e t} \sim 200$ microns is pumped through the channel at a time scale $\tau \sim \pi R_{\text {jet }}^{2} L_{j e t} / Q_{d}$. For a typical $Q_{d}=100 \mu \mathrm{L} / \mathrm{hr}, \tau \sim 0.8 \mathrm{~ms}$, longer

a

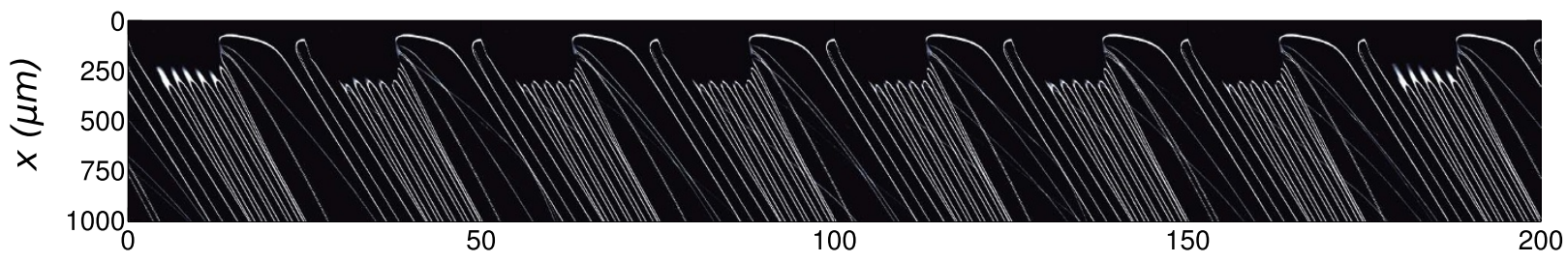

b

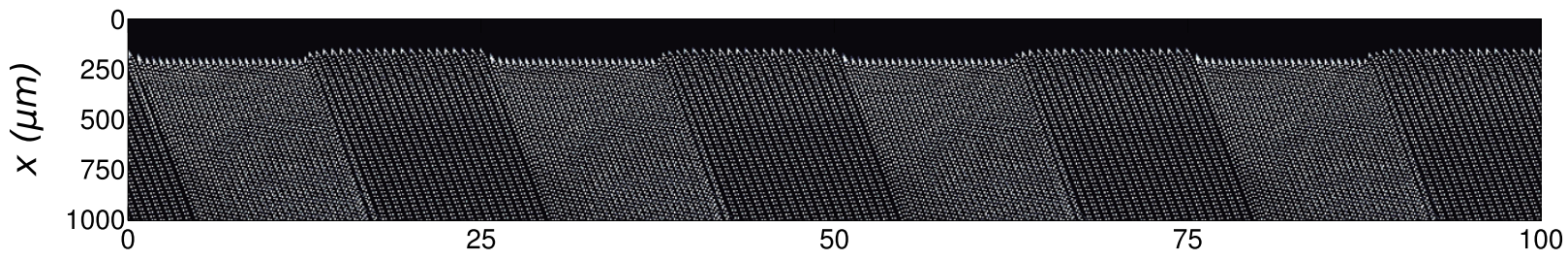

C

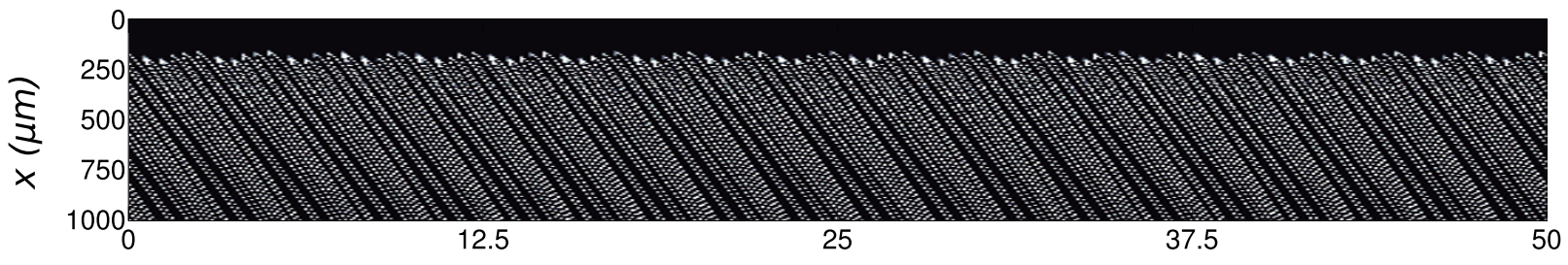

$t$ (ms)

Figure 3 Analysis of switching response time using space-time diagrams (using the center line of the channel, the black droplet interface being represented by the white lines). For 'low' flow rates $(75: 500 \mu \mathrm{L} / \mathrm{hr})$, the modulation frequency reaches the frequencies close to the droplet frequency in the absence of field (one or two large droplets produced, here the modulation is $40 \mathrm{~Hz}$, (a)). At 'high' flow rates (200: $1600 \mu \mathrm{L} / \mathrm{hr}$ ), modulation of $40 \mathrm{~Hz}$ are more easily visible (b) and up to $400 \mathrm{~Hz}$ modulation is obtained (c). The electric actuation provides fast switching between frequencies. 
a

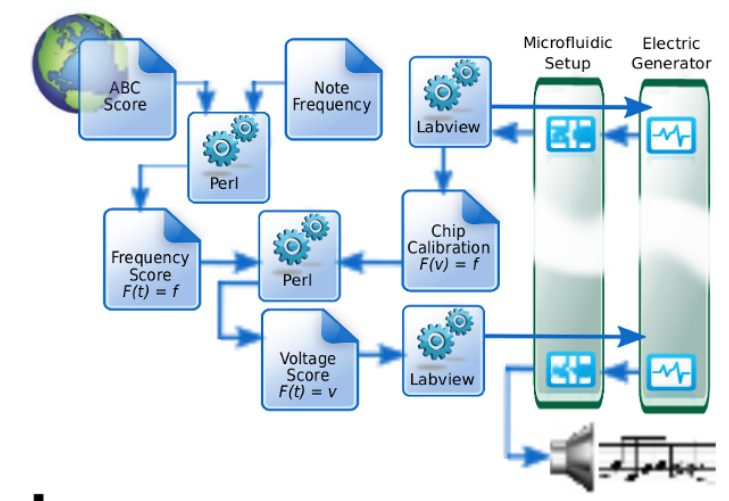

d

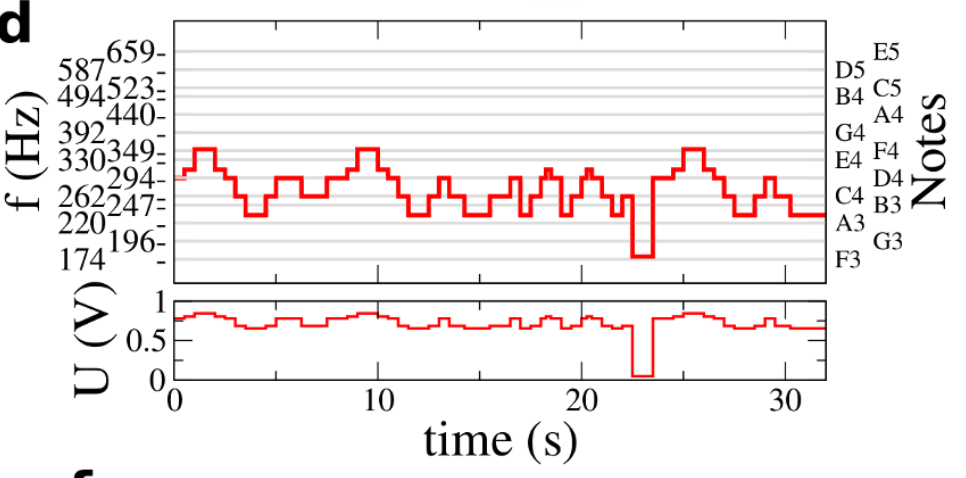

b
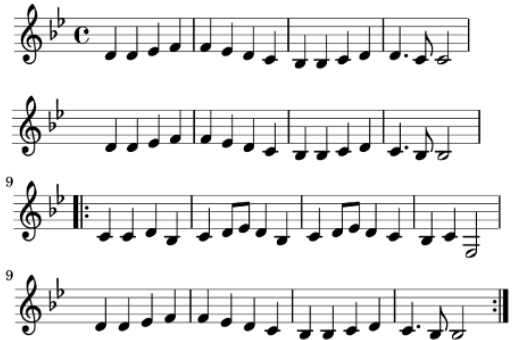

C

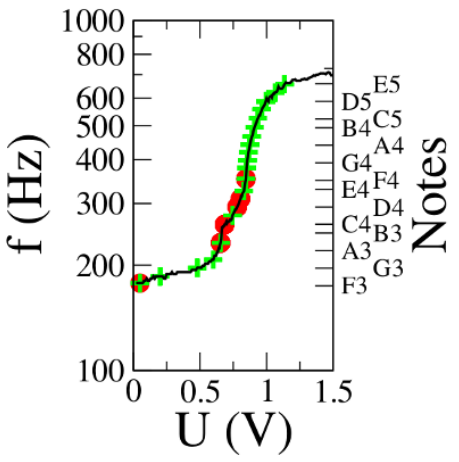

f
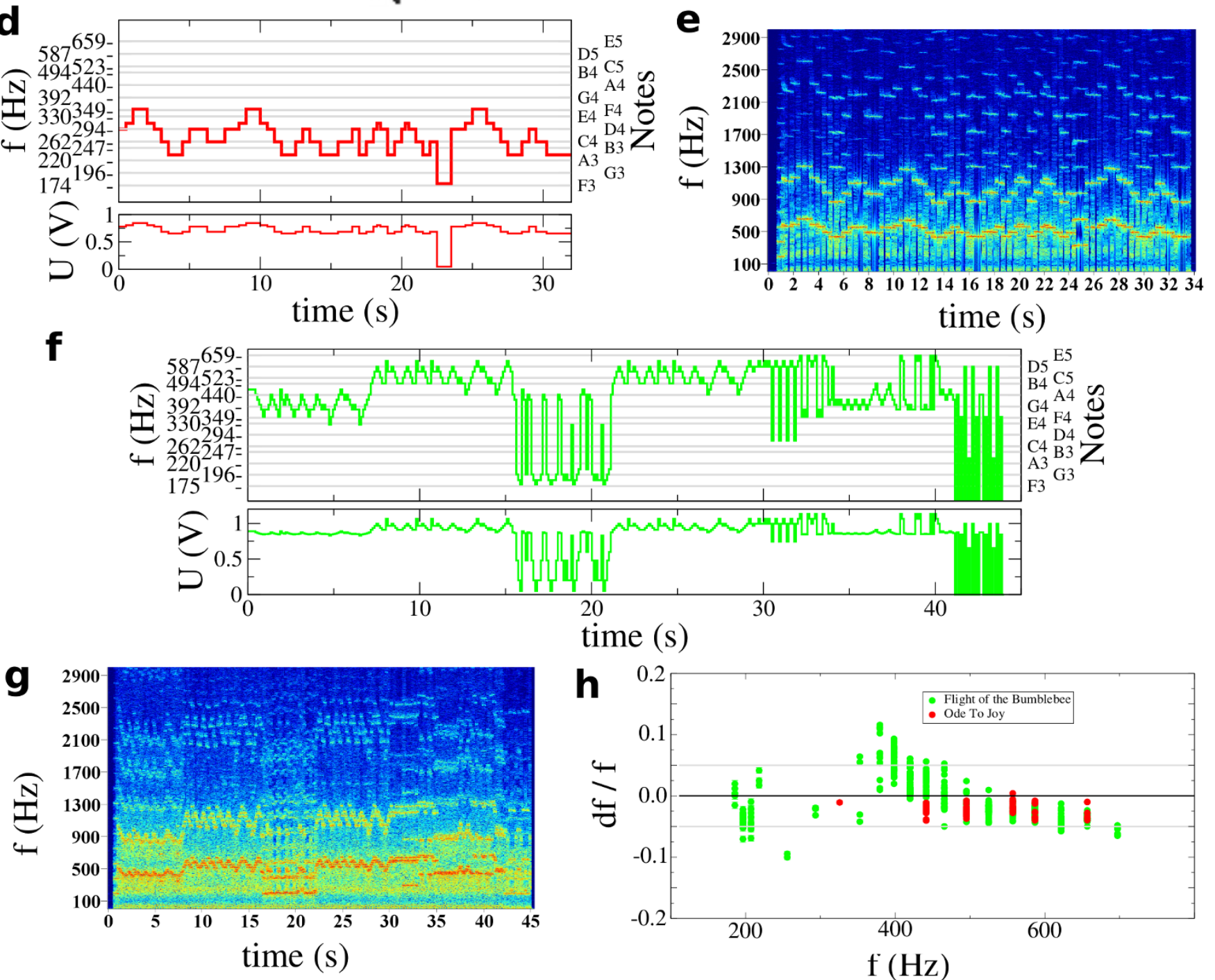

Figure $4 \mid$ The microfluidic Jukebox. (a) Scheme of the instrumentation process. A score, for example here Ode to Joy, Beethoven (b) is downloaded from the web. Based on the calibration curve of the chip (c) the score is converted and transposed using a Perl script in a series of frequencies accessible to the microfluidic chip (d). A Labview routine reads the series of frequencies to apply the corresponding voltage to the chip (see also Figure 2).

(e) The signals are recorded on an audio card and further analyzed to determine the frequency spectrum of the sound as a function of time. A similar scheme is used for The Flight of the Bumblebee, Korsakov (See Supplementary Figure 2), to determine the control voltages (f) and record and analyze the sound $(\mathrm{g})$. Finally, we analyze the measured frequency as a function of the target frequency and display the relative error $(\mathrm{d} f / f)$ between both. All notes are within less that 5\% error for the Ode to Joy. A larger error is made with The Flight of the Bumblebee as a result of the wider frequency range of the whole score, speed of actuation and step between notes. See also Supplementary Figure 4 for additional information and the Supporting audio and video files to listen to the sound of the microfluidic Jukebox. 
than $\tau_{\text {cap }}$. This would set a limit of actuation in the range of $1 \mathrm{kHz}$, close to the limit we reached here. This simple argument is recovered by considering that the capillary number is smaller than unity implying that the capillary speed $\eta / \gamma$ is smaller than the flow speed: the response of the fluid to a modification of surface tension is limited by the main flow. Interestingly, the period of droplet production reads $\tau_{\text {prod }} \sim \pi R_{\text {drop }}^{3} / Q_{d}$. Therefore the limit of actuation can be reexpressed as $\tau \sim \tau_{\text {prod }} L_{j e t} R_{\text {jet }}^{2} / R_{\text {drop }}^{3}$. For a given set of flow rate the actuation response time is of order the droplet production frequency, within a geometric correction $\gtrsim 1$ as we only observed jets and droplet sizes of order the channel size. Our system is therefore able to work at the single droplet level, until the hydrodynamic limits are reached (typically around $10 \mathrm{kHz}$ for our range of parameters).

The microfluidic Jukebox. Our approach provides controlled frequencies and fast switching. The last ingredient required to produce a sound track is the ability to create pauses between notes. As a zero frequency is not achievable in our system, we generate a pause by switching off the laser through electronic control. A note is then generated by fixing the applied voltage and turning the laser on for fluorescence excitation. This also enables to discriminate between two identical successive black notes and one single white note (for example, .) would sound like. ). This operation does not change the transition between different notes.

The sound-track is obtained from a midi file or abc score (www. abcnotation.com). We show here the example of the Beethoven $9^{\text {th }}$ Symphony (Ode to Joy, the anthem of the European Union) (Figure 4). We transform this music file in a frequency $v$ s. time file via an home made Perl program. Then the frequency sequence is converted into actuation voltages, based on the tuning of the microfluidic chip. In this operation, we take into account specific features of the device: at the dripping to jetting transition, the frequency can vary significantly for a small voltage variation. This effectively leads to a frequency range not reliably accessible. The Perl program provides means to adapt the piece to our instrument by shifting it by a certain number of half tones until all notes can be played. This operation is performed as a standard by musicians playing on an instrument a piece made for another one. Importantly, the forbidden gap is flow rate and system dependent. As a consequence, our instrument behaves as a standard musical instrument with specific features in terms of accessible frequencies. We finally use a Labview program to set the required voltage and the laser control in order to play the songs (Figure 4).

For Ode to Joy, the piece has been played for about $30 \mathrm{~s}$ with frequencies in the range $170-340 \mathrm{~Hz}$ (Figure 4, the sound and movie file is provided as Supplementary information). The notes are reliably reached within less than $5 \%$ error (roughly corresponding to one halftone). This typical error corresponds to the literature values obtained for dispersion of sizes in flow flocusing and accounts for all fluctuations present in the system. In order to push the limit of the system, we then demonstrated the capabilities of the system with a more demanding score, namely the Flight of the Bumblebee in which the switching between notes is much faster, with sharp frequency switches and a more extended range of notes. In this case again, the instrument follows the score as adapted based upon the calibration, showing the reliability of our instrument and in general of droplet actuation by electric fields. The target frequency displays a larger error (sometimes beyond 5\%) than for the Ode to Joy as a result of the constraints of the musical track. The maximum error is made when the system is close to the dripping to jetting transition as one would expect: at this transition point the system is very sensitive to small perturbation. However, all notes are played within a very good level of accuracy and further design optimization can in principle improve the system.

The short transition time between notes leads to a small 'glissando'. This 'glissando' which gives smooth transitions between notes could in principle also be programmed in our software by for example inducing smooth voltage transitions rather than sharp square as used here. The capabilities of this new instrument could in principle be further investigated by musicians; with the current state of the device, virtually all songs spanning one scale are accessible but specific features might be worth of interest to trained musicians.

\section{Discussion}

We propose here a microfluidic system usable to play music and as such our microfluidic chip can be considered as a novel music instrument combining fluids, light and electric phenomena and therefore branching a whole range of physical principles. Optimization of the device and of the sound recording would reduce in the long run the level of noise and more complex operations could also be implemented to produce polyphonic sound tracks (for example using different dyes and parallel droplet production units). Similarly, the intensity of the sound could be modulated either by a control of the fluorescein concentration or by a modulation of the laser power. Other effects could be obtained using a modulation of the laser intensity: (i) a 'tremolo' could be induced by small intensity modulation at a few Hertz, (ii) a 'vibrato' could be obtained by frequency modulation: a small modulation of the applied voltage at a few Hertz is technically accessible as shown in Fig. 3, (iii) a 'portamento' would be obtained with a smooth variation of the applied voltages similar to the 'glissando' mentioned previously; all these effect would provide a rich range of sounds. The tone of the instrument - i.e. the weight of the different harmonics in the spectrum - could also be modulated by changing the ratio of droplet size to laser spot size. These optimizations and improvements are of course of interest to generate more pleasing and rich musical tracks.

Our device is reminiscent of another system based on Minnaert resonance ${ }^{35}$. Minnaert investigated for the first time the sound of bubbles showing that bubbles have a resonance frequency, function of their size. He achieved controlled production of notes over one octave. Playing a song is achieved by generating bubbles of controlled sizes in the so-called bubble piano which makes use of this resonance. In contrast to this system, our approach does not rely on a resonance but on the instability of a liquid jet leading to periodic pinchoff at a fixed frequency. We do not measure here a pressure wave in the channel but a transduced signal of the corresponding frequency. However, the droplet production mechanism leads to pressure fluctuation at the nozzle linked to the Laplace pressure upon pinch-off as recently measured ${ }^{36}$. Such pressure fluctuations are of the order of \pm 0.01 bar at the production nozzle in droplet microfluidics during droplet production, and are a sound of the corresponding frequency. Such pressure waves could be amplified through resonators to be detected in the reverse approach to the one proposed earlier ${ }^{7}$.

A straightforward application in emulsion science is to listen to the sound produced to monitor how droplets are generated in microfluidics. As a frequency shift down to $1-3 \mathrm{~Hz}$ can be detected, one could in principle determine during droplet production if the production is changing over time. Applications in high-throughput screening could be the detection of hits in a population of fluorescent droplets. Droplets can be reinjected at frequencies reaching several thousands of droplets per second. If the droplets contain mainly inactive variants in an assay leading to a low fluorescent signal, a sound will be generated at a well defined frequency. A hit (a positive variant) would distort the signal for a few millisecond, leading to a signal detectable by an operator. Another application would be the creation of feedback loops that would stabilize the frequency of droplet production in emulsification. Indeed using a real time control of the applied voltage based on the time between successive droplets would enable specific frequencies to be reached without the need of an accurate calibration of the system. Controlled synchronization between different emulsion streams could then be achieved. Such control would however be made at the expense of a variation of volume of the droplets (as the remaining free parameter). 
In summary, we designed a microfluidic chip usable as a musical instrument. The key features are on the one hand the reliability of droplet production at a flow focusing junction and on the other hand, the electrical control of droplet production which is sufficiently fast to achieve a modulation of the droplet frequency down to a few milliseconds. Beyond the entertainment aspect, we believe that our system will be of interest in droplet-based microfluidics for the fine tuning of droplet size in given flow conditions. Such an option would be of interest for example for materials synthesis and control on chip when the flow conditions are not fully compatible with the physical properties of the materials (e.g. viscosity or surface tension). We finally hope that our approach will trigger initiatives towards the development of novel applications of micro and nanotechnologies.

\section{Methods}

Electric droplet microfluidic system. The electric droplet microfluidic system (Figure 1) is fabricated using standard photo-and soft-lithography techniques Rectangular electrodes with a separation distance of about $170 \mu \mathrm{m}$ are incorporated into the system by filing the microchannels with a metal alloy (Indalloy 19, Indium corporation). No alignment of the electrodes is required as the microchannels form the fluidic channels and the templates for the electrodes. Hence, only a single photomask is required in the fabrication process. A crossjunction microfluidic channel of $100 \mu \mathrm{m}$ width and depth $35 \mu \mathrm{m}$ is used to form droplets by flowing two immiscible fluids into separate microchannels at fixed flow rates via syringe pumps (neMESYS, Cetoni). The dispersed phase fluid flows in the center channel while the continuous phase fluids flow in the two side channels. Alternating ( $50 \mathrm{kHz}$ sine wave) voltages $u$ generated using a signal generator (33210A, Agilent) and amplified (to a $\mathrm{kV}$ voltage $U=490 \times u=0$ $1040 \mathrm{~V})$ using a high voltage amplifier $(623 \mathrm{~B}$, Trek) are then applied to the top pair of electrodes to control and manipulate the size of the droplets formed. The bottom pair of the electrodes and the ITO conductive electrode under the glass are connected to the ground.

Emulsification system. We produce Water-in-Oil (W/O) emulsions. For the dispersed phase $\left(Q_{d}\right)$ fluid, we use milli-Q water mixed with $10 \mu \mathrm{M}$ of Fluorescein (Sigma-Aldrich). For the continuous phase $\left(Q_{c}\right)$, we use mineral oil (M5904, from Sigma-Aldrich) with $5 \mathrm{wt} \%$ Span 80 (Sigma-Aldrich) as surfactant to prevent the coalescence of the droplets. The conductivity of the dispersed phase fluid is $2.2 \mu \mathrm{S} / \mathrm{cm}$ measured with a commercial conductivity meter (Eutech 2700, Thermo Fisher Scientific). The equilibrium surface tension of the oil water interface is $4-5 \mathrm{mN} / \mathrm{m}$ in the presence of $5 \%$ surfactant measured in using the pendant droplet method (Sinterface PAT1)

Optical setup set up. The fluorescence set up is sketched in Supplementary Figure 1. The set-up is similar to previously described systems ${ }^{20}$. The chips are mounted on a Olympus IX81 Microscope equipped with a MBL $473 \mathrm{~nm}$ laser at $20 \mathrm{~mW}$ output power. The laser is reflected by a dichroic mirror and focused through the microscope objective into the channel. A droplet containing fluorescein emits a fluorescence light when flowing through the laser. The fluorescent signal is transmitted by the dichroic which also reflects the backscattered laser light. A notch filter absorbs the remaining scattered light in order to keep only the fluorescence signal. This filtered signal is then reflected on a second dichroic towards a photomultiplier (PMT), powered at $\pm 15 \mathrm{~V}$ (ALR 3003 ELC). The PMT gain is controlled by a 0.1-0.4 V voltage source (AL841B ELC), or alternatively by a Labview input/output card and homemade routine. A typical PMT signal is shown in Figure 1. The PMT output is connected to the computer through the audio card which digitalized the signal (AD converter; $44.1 \mathrm{kHz}$ sampling rate and 16 bits depth). Bright field high-speed imaging is performed using a Phantom V311 camera.

Data analysis. The frequency contents of the signal is extracted using a Fast Fourier Transform (FFT) algorithm based on 16384 samples, which enable to obtain a spectrogram with a definition of $2.7 \mathrm{~Hz}$. The droplet production frequency is then estimated by extracting the first partial (fundamentals)

of the recorded PMT signal with the resolution limit of $2.7 \mathrm{~Hz}$. In the music tracks, we also extract the sound frequency by measuring the spacing between droplets.

Playing music. The chip is calibrated to determine droplet production frequency as a function of applied voltage. Musical scores are obtained from www.abcnotation.com. A perl program converts the scores into series of frequencies based on the equal temperament scale (Supplementary Table 1). The score is adapted by shifting it by an integer number of half tones to fit the accessible range of frequencies.

1. Riedel-Kruse, I. H., Chung, A. M., Dura, B., Hamilton, A. L. \& Lee, B. C. Design, engineering and utility of biotic games. Lab Chip. 11, 14-22 (2011).

2. Khatib, F. et al. Crystal structure of a monomeric retroviral protease solved by protein folding game players. Nat. Struct. Mol. Biol. 18, 1175-1177 (2011).
3. Hennig, H. et al. The nature and perception of fluctuations in human musical rhythms. PLOS one. 6, e26457 (2011).

4. Boyd, J. W. R. \& Varley, J. The use of passive measurement of acoustic emissions from chemical engineering processes. Chem. Eng. Sci. 56, 1749-1767 (2011).

5. Franke, T., Abate, A. R., Weitz, D. A. \& Wixforth, A. Surface acoustic wave (SAW) directed droplet flow in microfluidics for PDMS devices. Lab Chip. 9, 2625-2627 (2009).

6. Franke, T., Braunmüller, S., Schmid, L., Wixforth, A. \& Weitz, D. A. Surface acoustic wave actuated cell sorting (SAWACS). Lab Chip. 10, 789-794 (2010).

7. Langelier, S. M., Chang, D. S., Zeitoun, R. I. \& Burns, M. A. Acoustically driven programmable liquid motion using resonance cavities. Proc. Natl. Acad. Sci. U.S.A. 106, 12617-12622 (2009).

8. Xia, Y. N. \& Whitesides, G. M. Soft lithography. Annu. Rev. Mater. Sci. 28, 153-184 (1998)

9. Whitesides, G. M. et al. Lab on a chip. Nature. 442, 367-418 (2006).

10. Teh, S. Y., Lin, R., Hung, L.-H. \& Lee, A. P. Droplet microfluidics. Lab Chip. 8, 198-220 (2008).

11. Theberge, A. B. et al. Microdroplets in microfluidics: An evolving platform for discoveries in chemistry and biology. Angew. Chem., Int. Ed. 49, 5846-5868 (2010)

12. Guo, M. T., Rotem, A., Heyman, J. A. \& Weitz, D. A. Droplet microfluidics for high-throughput biological assays. Lab Chip. 12, 2146-2155 (2012).

13. Agresti, J. J. et al. Ultrahigh-throughput screening in drop-based microfluidics for directed evolution. Proc. Natl. Acad. Sci. U. S. A. 107, 4004-4009 (2010).

14. Miller, O. J. et al. High-resolution dose-response screening using droplet-based microfluidics. Proc. Natl. Acad. Sci. U.S.A. 109, 378-383 (2012).

15. Pekin, D. et al. Quantitative and sensitive detection of rare mutations using droplet-based microfluidics. Lab Chip. 11, 2156-2166 (2011).

16. Seemann, R., Brinkmann, M., Pfohl, T. \& Herminghaus, S. Droplet-based microfluidics. Rep. Prog. Phys. 75, 016601 (2012).

17. Frenz, L. et al. Droplet-based microreactors for the synthesis of magnetic iron oxide nanoparticles. Angew. Chem., Int. Ed. 47, 6817-6820 (2008).

18. Abate, A. R., Hung, T., Mary, P., Agresti, J. J. \& Weitz, D. A. High-throughput injection with microfluidics using picoinjectors. Proc. Natl. Acad. Sci. U. S. A. 107, 19163-19166 (2010)

19. Fallah-Araghi, A., Baret, J.-C., Ryckelynck, M. \& Griffiths, A. D. A completely in vitro ultrahigh-throughput droplet-based microfluidic screening system for protein engineering and directed evolution. Lab Chip. 12, 882-891 (2012).

20. Baret, J.-C. et al. Fluorescence-activated droplet sorting (FADS): efficient microfluidic cell sorting based on enzymatic activity. Lab Chip. 9, 1850-1858 (2009).

21. Abate, A. R. \& Weitz, D. A. Single-layer membrane valves for elastomeric microfluidic devices. Appl. Phys. Lett. 92, 243509 (2008).

22. Tan, S. H., Nguyen, N.-T., Yobas, L. \& Kang, T. G. Formation and manipulation of ferrofluid droplets at a microfluidic T-junction. J. Micromech. Microeng. 20, 045004 (2010).

23. Tan, S.-H., Semin, B. \& Baret, J.-C. Microfluidic flow focusing in ac electric field. Lab Chip. 14, 1099-1106 (2014).

24. Malloggi, F., Gu, H., Banpurkar, A. G., anapalli, V. S. A. \& Mugele, F. Electrowetting -a versatile tool for controlling microdrop generation. Eur. Phys. J. E Soft Matter. 26, 91-96 (2008).

25. Anna, S. L., Bontoux, N. \& Stone, H. A. Formation of dispersions using "flow focusing" in microchannels. Appl. Phys. Lett. 82, 364-366 (2003).

26. Cubaud, T. \& Mason, T. G. Capillary threads and viscous droplets in square microchannels. Phys. Fluids. 20, 053302 (2008).

27. Nunes, J. K., Tsai, S. S. H., Wan, J. \& Stone, H. A. Dripping and jetting in microfluidic multiphase flows applied to particle and fiber synthesis. J. Phys. D: Appl. Phys. 46, 114002 (2013).

28. Utada, A. S., Fernandez-Nieves, A., Stone, H. A. \& Weitz, D. A. Dripping to jetting transitions in coflowing liquid streams. Phys. Rev. Lett. 99, 094502 (2007).

29. Utada, A. S., Fernandez-Nieves, A., Gordillo, J. M. \& Weitz, D. A. Absolute instability of a liquid jet in a coflowing stream. Phys. Rev. Lett. 100, 014502 (2008)

30. Baret, J.-C., Beck, Y., Billas-Massobrio, I., Moras, D. \& Griffiths, A. D. Quantitative cell-based reporter gene assays using droplet-based microfluidics. Chem. Biol. 17, 528-536 (2010)

31. Harry, F. Olson. Music, Physics and Engineering. Dover Publications, 1967

32. Kieffer, C. A. et al. A high-precision fluid handling system based on pressure actuation: multi-inlets flow rate control. Proc. 3rd Eur. Conf. Microfluidics. 252 (2012).

33. Abate, A. R., Thiele, J., Weinhart, M. \& Weitz, D. A. Patterning microfluidic device wettability using flow confinement. Lab Chip. 10, 1774-1776 (2010).

34. Baret, J.-C., Decré, M. M. J., Herminghaus, S. \& Seemann, R. Electroactuation of fluid using topographical wetting transitions. Langmuir. 21, 12218-12221 (2005).

35. Minnaert, M. On musical air-bubbles and the sound of running water. Phil. Mag 16, 235-248 (1933)

36. Romero, P. A. \& Abate, A. R. Flow focusing geometry generates droplets through a plug and squeeze mechanism. Lab Chip. 12, 5130-5132 (2012).

\section{Acknowledgments}

The authors thank Valérie Taly (Université Paris Descartes), Jörg Enderlein (Universität Göttingen) and Stephan Herminghaus (MPI-ds Göttingen) for insightfull discussions. The authors acknowledge the financial support of the Max-Planck Society and the support by a 
funding from the European Research Council (ERC) under the European Union's Seventh Framework Program (FP7/2007-2013)/ERC Grant agreement 306385-SofI. S.H.T. also acknowledges support from the GGNB doctoral school.

\section{Author contributions}

S.H.T. and F.M. performed the experiments and collected the data. B.S. contributed in analytical tools. J.V. designed the software and the tools for sound recording and analysis. J.C.B. conceived the project, supervised the experiments and analyzed the data. All authors contributed to the manuscript writing.

\section{Additional information}

Supplementary information accompanies this paper at http://www.nature.com/ scientificreports
Competing financial interests: A patent application with Priority date March 31st 2012 has been filled for the control of droplet production in electric fields.

How to cite this article: Tan, S.H., Maes, F., Semin, B., Vrignon, J. \& Baret, J.-C. The Microfluidic Jukebox. Sci. Rep. 4, 4787; DOI:10.1038/srep04787 (2014).

cc) (i) (2) This work is licensed under a Creative Commons Attribution-NonCommercialShareAlike 3.0 Unported License. The images in this article are included in the article's Creative Commons license, unless indicated otherwise in the image credit; if the image is not included under the Creative Commons license, users will need to obtain permission from the license holder in order to reproduce the image. To view a copy of this license, visit http://creativecommons.org/licenses/by-nc-sa/3.0/ 